\title{
Emergent motion of condensates in mass-transport models
}

\author{
Ori Hirschberg, ${ }^{1}$ David Mukamel, ${ }^{1}$ and Gunter M. Schütz ${ }^{2}$ \\ ${ }^{1}$ Department of Physics of Complex Systems, Weizmann Institute of Science, 76100 Rehovot, Israel \\ ${ }^{2}$ Institute of Complex Systems II, Forschungszentrum Jülich, 52425 Jülich, Germany
}

(Received 17 December 2012; published 13 May 2013)

\begin{abstract}
We examine the effect of spatial correlations on the phenomenon of real-space condensation in driven masstransport systems. We suggest that in a broad class of models with a spatially correlated steady state, the condensate drifts with a nonvanishing velocity. We present a robust mechanism leading to this condensate drift. This is done within the framework of a generalized zero-range process (ZRP) in which, unlike the usual ZRP, the steady state is not a product measure. The validity of the mechanism in other mass-transport models is discussed.
\end{abstract}

DOI: 10.1103/PhysRevE.87.052116

PACS number(s): 05.70.Ln, 02.50.Ey, 05.40.-a, 64.60.-i

\section{INTRODUCTION}

Nonequilibrium condensation, whereby a macroscopic fraction of microscopic constituents of a system accumulates in a local region, is a common feature of many masstransport systems. Examples include shaken granular gasses [1], vehicular traffic [2-4], the macroeconomics of wealth distribution [5,6], and others [7,8]. Mechanisms that can lead to the formation of condensates have been studied extensively in recent years, mainly by analyzing prototypical toy models. A primary role in these studies was played by the zero-range process (ZRP), an exactly solvable model in which particles hop between sites with rates that depend only on the number of particles in the departure site [9-11]. Extensions and variations of the ZRP have been used to study the emergence of multiple condensates [12], first-order condensation transitions [13,14], and the effect of interactions [15] and disorder [16] on condensation. Moreover, one-dimensional phase separation transitions in exclusion processes and other driven diffusive systems can quite generally be understood by a mapping on ZRPs [17].

The dynamics of condensates is less well explored. In the ZRP, where condensation takes place when a macroscopic fraction of particles occupies a single site, the resulting condensate does not drift in the thermodynamic limit [14,18-20]. It is shown below that this is related to the fact that the steady state of the ZRP is a product measure. In some real-world systems, however, condensates are in continual motion. For example, traffic jams, which can be viewed as condensates, are known to propagate along congested roads $[11,21,22]$. Recently, two variants of the ZRP were also found to relax to a time-dependent state in which the condensate performs a drift motion: one is a ZRP with non-Markovian hopping rates [23,24], and the other is a model with "explosive condensation" [25]. To date, there is no systematic understanding of the mechanism by which a macroscopic condensate motion emerges from the underlying nonequilibrium microscopic dynamics.

In this paper, we study how spatial correlations in the steady states may lead the condensate to drift with a nonvanishing velocity. We do so by introducing a generalization of the ZRP whose steady state does not factorize. Within its framework, we identify the mechanism that generates the drift. The analysis is based on numeric simulations and on a mean-field (MF) approximation which captures the essential effect of correlations in the condensed phase, and thus elucidates the different observed modes of condensate motion. The drift mechanism that we identify is robust and therefore it is expected to be valid in a broad class of spatially correlated mass-transport systems.

\section{MODEL}

We focus on a class of stochastic one-dimensional models defined on a ring with $L$ sites. At any given time, each site $i$ is occupied by $n_{i}$ particles with $\sum_{i} n_{i}=N, n_{i} \geqslant 0$. The model evolves by a totally asymmetric hopping process whereby particles hop from site $i$ to $i+1$ with a rate that depends on the occupation numbers $n_{i}$ and $n_{i-1}$. This is a generalization of the usual ZRP in which the rate depends only on $n_{i}$. More specifically, we choose the hopping rates to be of the form

$$
n_{i-1}, n_{i}, n_{i+1} \stackrel{w\left(n_{i-1}\right) u\left(n_{i}\right)}{\longrightarrow} n_{i-1}, n_{i}-1, n_{i+1}+1,
$$

with rates

$$
u\left(n_{i}\right)=1+\frac{b}{n_{i}}, \quad w\left(n_{i-1}\right)=\left\{\begin{array}{ll}
1 & n_{i-1} \neq 0 \\
\alpha & n_{i-1}=0
\end{array} .\right.
$$

The particular form of $u(n)$ is motivated by the fact that in the usual ZRP, which corresponds to $\alpha=1$, this choice with $b>2$ leads to a condensation transition [26]. The rate $w$ with $\alpha \neq 1$ represents an interaction between nearest-neighbor sites. According the dynamical rules (1) and (2), at every short time interval $d t$, each site $i$ whose occupation $n_{i} \geqslant 1$ may eject a particle with a probability $u\left(n_{i}\right) d t$, as long as the preceding site $(i-1)$ is occupied. If the preceding site is empty, this probability changes to $\alpha u\left(n_{i}\right) d t$. The model has three parameters: $b, \alpha$, and the density $\rho \equiv N / L$, which is conserved by the dynamics.

In the usual ZRP (the case of $\alpha=1$ ), the stationary distribution is known to factorize into a product of single site terms, and so can be exactly calculated $[9,27,28]$. This factorization property renders the ZRP quite special, as slight variations of the ZRP dynamics result in nonfactorizable models. To probe the effect of spatial correlations on the condensate, we choose for simplicity $w$ to be of the form (2), which leads to a spatially correlated steady state when $\alpha \neq 1$. The same drift motion which is described below is also found for other forms of $w(n)$, such as cases where $w(n) \neq 1$ 

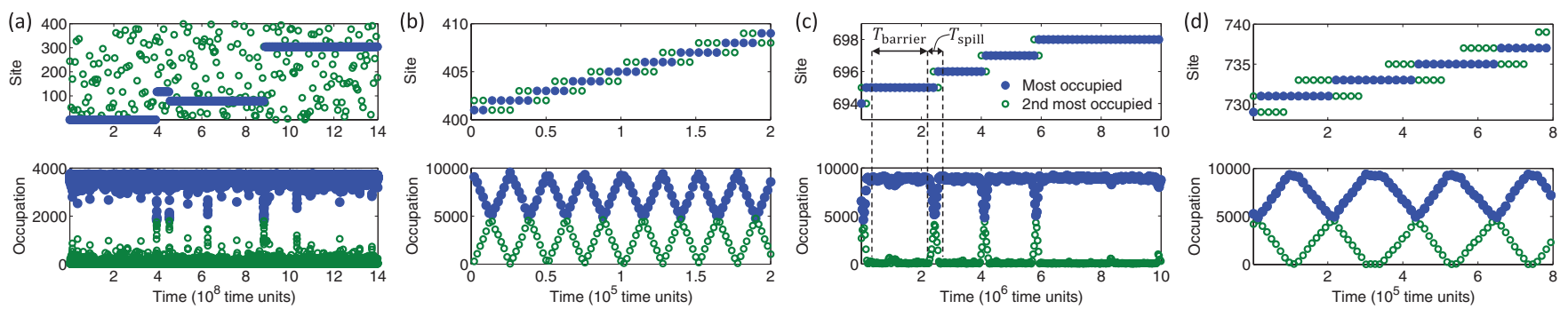

FIG. 1. (Color online) The location (top) and occupation (bottom) of the most occupied ( $\bullet$ ) and second most occupied ( $\circ$ ) sites for several values of $\alpha$. (a) $\alpha=1$, i.e., the usual ZRP. The condensate is stable for long times, and relocates to random distant sites. (b) $\alpha=1.5$. The condensate advances through a slinky motion from one site to the next. (c) $\alpha=1.05$. The condensate remains on each site for a long time before "spilling" to the next. The definitions of $T_{\text {barrier }}$ and $T_{\text {spill }}$ are indicated. (d) $\alpha=0.5$. The condensate skips every other site. In all cases $b=3, \rho=10$, and $L=1000$, except (a) where $L=400$. Note the different time scales.

for finitely many values of $n$, and for other choices of $u(n)$ which give rise to condensation [29].

The model (1) and (2) exhibits, as is evident in numerical simulations, a condensation transition with a drifting condensate. We have carried out Monte Carlo simulations of the model for several values of $\alpha$ in a system of size $L=1000$ and density $\rho=10$. After the system has relaxed to its steady state, the dynamics of the condensate was examined by tracking the position of the most occupied sites over time. The results are presented in Fig. 1 and in videos in the Supplemental Material [30]. In the usual asymmetric ZRP [Fig. 1(a)], it is known that the condensate is static up to time scales of order $L^{b}$ and then it relocates to a random site due to fluctuations [14,18-20]. There is a striking qualitative difference in the dynamics of model (1) and (2) when $\alpha \neq 1$ [Figs. 1(b)-1(d)], where the condensate is clearly seen to drift along the lattice. The condensate is seen to move from one site to the next when $\alpha>1$ [Fig. 1(b)], or to skip every other site when $\alpha<1$ [Fig. 1(d)]. In both cases, when $\alpha$ is not too close to 1 the motion is slinkylike, with the condensate "spilling" from an old site to a new one immediately after the previous spilling has completed. The drift becomes somewhat less regular when $\alpha$ is close to 1 . In this regime, the slinky motion is interrupted by periods of time when the condensate occupies a single site, before the spilling process is initiated [Fig. 1(c)]. This, however, is argued below to be a crossover mode, and the interval in $\alpha$ in which it is observed shrinks in the large $L$ limit.

\section{MODEL ANALYSIS}

\section{A. Mean-field approximation}

To understand these results we propose a mean-field analysis of the model in which the occupations of all sites are considered independent, but might not be identically distributed. Within this approximation, the current that arrives into site $i$ from site $i-1$ is a Poisson process whose rate we denote by $J_{i}$. The probability $P_{i}\left(n_{i}\right)$ to find $n_{i}$ particles in site $i$ thus evolves according to

$$
\begin{aligned}
\frac{d P_{i}\left(n_{i}\right)}{d t}= & P_{i}\left(n_{i}-1\right) J_{i}+P_{i}\left(n_{i}+1\right)\left\langle w_{i}\right\rangle u\left(n_{i}+1\right) \\
& -P_{i}\left(n_{i}\right)\left(J_{i}+\left\langle w_{i}\right\rangle u\left(n_{i}\right)\right),
\end{aligned}
$$

where

$$
\left\langle w_{i}\right\rangle \equiv \sum_{n=0}^{\infty} P_{i-1}(n) w(n)=1+(\alpha-1) P_{i-1}(0)
$$

encodes the mean effect of site $i-1$ on the hopping rate out of site $i$. Equation (3) is valid also when $n_{i}=0$ with the definition $P_{i}(-1) \equiv 0$. Equations (3) and (4) are to be solved with the self consistency condition $J_{i+1}=\sum_{n} P_{i}(n)\left\langle w_{i}\right\rangle u(n)$.

At low density, the system is in a subcritical, disordered phase (this will be shown below). In this homogeneous phase, $P_{i}(n)=P(n)$ and $J_{i}=J$ for all sites $i$. At higher densities, however, condensation takes place, where the translational symmetry is spontaneously broken and both $P_{i}(n)$ and $J_{i}$ depend on the distance of site $i$ from the condensate. This dependence of $P$ and $J$ on $i$ is a result of the correlations that exist in the steady state of the model, and it provides the mechanism for the condensate drift: a nonhomogeneous $J_{i}$ implies that in some sites the outflowing current is smaller than the incoming current, leading these sites to accumulate particles while other sites are similarly being depleted of particles. We shall now demonstrate that this occurs in our model.

In the homogeneous (subcritical and critical) phases, the model eventually reaches a steady state. In the nonhomogeneous supercritical phase, however, the condensate location keeps moving with time. The analysis of this time-dependent phase is based on one key observation: the time scale of the microscopic dynamics, which for the rates (2) is of order 1, is much faster than that of the condensate motion. As shown below, the time scale of the spilling process is of order $L$, validating this observation in the thermodynamic limit. Due to this time-scale separation, while the condensate (i.e., the most occupied site) is static all other sites reach a quasistationary distribution.

In both phases, by equating the LHS of Eq. (3) to zero the (quasi)stationary distribution is found to be

$$
P_{i}(n)=P_{i}(0) z_{i}^{n} \prod_{k \leqslant n} \frac{1}{u(k)}, \quad \text { with } \quad z_{i} \equiv J_{i} /\left\langle w_{i}\right\rangle .
$$

Here, $z_{i}$ plays the role of a "fugacity" of site $i$. For rates of the form (2), the normalization of $P_{i}(n)$ yields

$$
P_{i}(0)=\left[{ }_{2} F_{1}\left(1,1 ; b+1 ; z_{i}\right)\right]^{-1},
$$


where ${ }_{2} F_{1}$ is a hypergeometric function [note that $P_{i}(0)$ depends on the exact form of $u(n)$ and not just on its large $n$ asymptotics]. The occupation probability is asymptotically given by $P_{i}(n) \sim n^{-b} z_{i}^{n}$.

We first examine the solution (5) in the subcritical and critical phases, and show that the model undergos a condensation transition. Since the system is homogenous in these phases, the subscript $i$ may be dropped from Eqs. (4)-(6). The fugacity can now be determined in terms of the density by inverting the relation

$$
\rho(z)=\sum_{n} n P(n)=\frac{{ }_{2} F_{1}(2,2 ; b+2 ; z)}{(1+b)_{2} F_{1}(1,1 ; b+1 ; z)} z,
$$

where the RHS is obtained by substituting Eq. (5) in the sum. Similarly, Eq. (4) for $\langle w\rangle$ reads in the homogeneous phases $\langle w\rangle=1+(\alpha-1)\left[{ }_{2} F_{1}(1,1 ; b+1 ; z)\right]^{-1}$.

The density (7) is an increasing function of $z$ that attains its maximum at $z=1$, which is its radius of convergence about the origin. A finite density at $z=1$ indicates a condensation phase transition, which is mathematically similar to BoseEinstein condensation [9]. By substituting $z=1$ in (7) it is seen that condensation takes place when $b>2$, in which case the critical density is $\rho_{c}=1 /(b-2)$, the same value as that of the usual ZRP. The critical current is similarly found to be $J_{c}=\langle w\rangle_{z \rightarrow 1}=1+(\alpha-1) b /(b-1)$. As long as $\rho<\rho_{c}$, the system remains in a homogeneous subcritical phase. When $\rho$ is increased, the current $J$ increases until $\rho$ and $J$ reach their critical values and all sites of the system are in a homogenous critical phase. When the density is further increased, condensation sets in, breaking the translational invariance of the system.

Let us now discuss the nonhomogeneous supercritical phase and the mechanism of the condensate motion. We focus on the case of $\alpha>1$. In this case, the condensed phase is composed of a condensate, which at any given time consists of two macroscopically occupied consecutive sites (say 1 and 2), while the rest of the sites are microscopically occupied. This differs from the usual ZRP where the condensate is typically supported by a single site. We show that $J_{2}>1$ and $J_{i}=1$ for $i \neq 2$. This results in an increase of the occupation of site 2 at the expense of site 1 over a macroscopic $O(L)$ time scale, while the rest of the sites are in a quasistationary state. Therefore, the condensate drifts with a velocity of order $L^{-1}$

The analysis begins at site 1 , whose occupation we assume is $n_{1}=O(L) \gg 1$, and thus it emits a mean current $J_{2}=$ $\left\langle w_{1}\right\rangle\left(1+\left\langle b / n_{1}\right\rangle\right) \simeq\left\langle w_{1}\right\rangle$. At the moment, $\left\langle w_{1}\right\rangle$ is unknown. It is determined self-consistently at the end of the calculation. Since $P_{L}(0) \neq 0$, as is established below, it is seen that $J_{2}>1$ (since $\alpha>1$ ). We now proceed to examine the second site. As long as site 1 accommodates the condensate it is never empty, i.e., $P_{1}(0)=0$. It follows from (4) that $\left\langle w_{2}\right\rangle=1$. The fugacity of the second site is then $z_{2} \equiv J_{2} /\left\langle w_{2}\right\rangle \simeq J_{2}>1$, and therefore its occupation distribution (5) cannot be normalized. This means that as long as site 1 is highly occupied, site 2 tends to accumulate particles, implying that its occupation too becomes macroscopic (of order $L$ ) for a long period of time [31,32]. We call such a site with fugacity $z_{i}>1$ supercritical.
The analysis now continues site by site in a similar fashion. For each site $i,\left\langle w_{i}\right\rangle$ is calculated using (4) from the known value of $P_{i-1}(0)$. The fugacity of site $i(5)$ is then calculated from $\left\langle w_{i}\right\rangle$ and the incoming current into the site $J_{i}$. Once the fugacity is known, $P_{i}(0)$ and $J_{i+1}$ are determined from (6) and from $J_{i+1}=\sum_{n} P_{i}(n)\left\langle w_{i}\right\rangle u(n)$, and the process is repeated in the next site. Performing this analysis reveals that site 3 is critical (i.e., $z_{3}=1$ ) and sites $i=4, \ldots, L$ are subcritical $\left(z_{i}<1\right)$, with $J_{i}=1$ and $\left\langle w_{i+1}\right\rangle=1+(\alpha-$ $1) / 2 F_{1}\left(1,1 ; b+1 ; 1 /\left\langle w_{i}\right\rangle\right)$ for all $i \geqslant 3$. This recursion relation defines a sequence $\left\langle w_{i}\right\rangle$, which converges (exponentially rapidly) to a unique fixed point $w^{*}(\alpha, b)$, which is the solution of the equation

$$
w^{*}=1+\frac{\alpha-1}{{ }_{2} F_{1}\left(1,1 ; b+1 ; 1 / w^{*}\right)},
$$

and thus satisfies $w^{*}(\alpha, b)>1$ for all $\alpha>1$. When $L \gg 1$, the periodic boundary conditions imply that $\left\langle w_{1}\right\rangle \simeq w^{*}>1$, and thus Eq. (5) confirms that $P_{L}(0)>0$. This closes the loop self-consistently and completes the calculation of the quasistationary distribution for the nonhomogenous phase.

A natural order parameter for the condensation transition is the bulk density of the "background fluid" $\rho_{\mathrm{BG}}$, which can be defined as the mean density of all but the two most occupied sites (since the condensate is typically carried by two sites). Below the transition, $\rho_{\mathrm{BG}}=\rho$, which approaches $\rho_{c}=1 /(b-2)$ as the transition is approached from below. Above the transition, all sites outside of a finite boundary layer around the condensate are subcritical with a mean occupation of $\rho_{\mathrm{BG}} \simeq \rho\left(z=1 / w^{*}\right)<\rho(1)=\rho_{c}$ since the function $\rho(z)$, Eq. (7), increases monotonically with $z$. Therefore, the condensation transition is found to be a discontinuous (first order) one. This is in contrast to the usual ZRP with rates (2) and $\alpha=1$ where the transition is continuous. A similar discontinuity exists in the current, which jumps from $J_{c}>1$ just below $\rho_{c}$ to $J=1$ just above it.

We now discuss the emergent dynamics of the condensate and identify two distinct modes of motion: a regular slinky motion, and an irregular motion through a barrier. The motion of the condensate from one site to the next consists of two stages: a "spilling" stage during which it is supported on two sites, and a period before this spilling is initiated, when the condensate is carried by a single site. We first consider the spilling process. According to the calculation above, the number of particles that accumulate in the second condensate site per unit time is on average $J_{2}-J_{3}=w^{*}-1$. As there are $N_{\text {cond }} \simeq\left(\rho-\rho_{\mathrm{BG}}\right) L$ particles in the condensate, the total spilling time $T_{\text {spill }}$ scales, to leading order, linearly with the system size: $T_{\text {spill }}=\left(\rho-\rho_{\mathrm{BG}}\right) L /\left(w^{*}-1\right)$. This justifies the assumption of time-scale separation which underlies the existence of a quasistationary state. The condensate velocity equals $T_{\text {spill }}^{-1}$, and therefore scales as $L^{-1}$. In the limit of $\alpha \rightarrow 1$, the spilling time diverges.

Once a spilling is complete, there is a moment that the condensate is located solely on a single site. We now relabel this site as site 1 . At this moment, the occupation of the following site is $n_{2} \approx \rho_{\mathrm{BG}}=O(1)$. The rate at which particles leave the second site is, at this stage, approximately $J_{3} \approx 1+b / \rho_{\mathrm{BG}}$, which should be compared with the rate of incoming particles, $J_{2} \simeq w^{*}$. According to Eq. (8) and the definition of $\rho_{\mathrm{BG}}$, the 
two rates are equal when $\alpha=\alpha^{*}$ which is the solution of the equation $\alpha^{*}=1+b_{2} F_{1}\left(1,1 ; b+1 ; 1 / w^{*}\left(b, \alpha^{*}\right)\right) / \rho_{\mathrm{BG}}\left(b, \alpha^{*}\right)$. The mode of condensate motion now depends on whether $\alpha$ is larger or smaller than $\alpha^{*}$.

(i) When $\alpha>\alpha^{*}$, the initial current into site 2 is larger than the mean current out of this site, and a spilling of the condensate is initiated immediately. In this case, the condensate drifts continuously in a slinky motion as in Fig. 1(b).

(ii) On the other hand, $J_{2}<J_{3}$ when $1<\alpha<\alpha^{*}$, and thus particles do not immediately accumulate on site 2 . The incoming current into the site surpasses the outgoing current and spilling sets in only after fluctuations bring the occupation of the second site to a value $n^{*}(\alpha, b)$ which is defined by $w^{*}=1+b / n^{*}$. The ensuing motion of the condensate is more irregular, with a stable condensate, which occasionally spills to the next site as in Fig. 1(c). Note that the mean time $T_{\text {barrier }}$ it takes before a fluctuation brings $n_{2}$ over the barrier $n^{*}$ does not scale with the system size. Thus, in a large enough system the condensate regularly drifts and is typically supported by two neighboring sites for any value of $\alpha>1$.

\section{B. Numerical results}

The numerical simulations support the qualitative picture that emerges from the MF analysis presented above. The existence of two modes of motion, slinkylike and irregular, and the crossover between them as $\alpha$ is increased conform with numerical findings (Fig. 1). In particular, the spilling mechanism between the two condensate sites in which the accumulation of particles is linear in time is verified [Fig. 1(b)], thus also implying that the drift velocity scales as $L^{-1}$ as predicted. Furthermore, the first-order nature of the transition, as manifested by the $\rho_{\mathrm{bg}}(\rho)$ curve, and the subcritical nature of the background fluid are presented in Fig. 2.

As expected, there are quantitative differences between the the MF predictions and numerical results. For example, for $b=3$ and $\alpha=1.5$, the simulation values of the critical

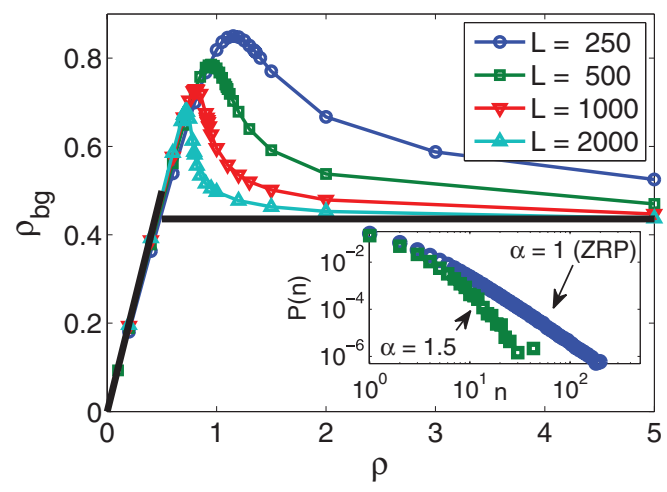

FIG. 2. (Color online) A first-order phase transition is seen in the background density $\rho_{\mathrm{BG}}$ as a function of the density $\rho$. Numerical results for several system sizes are plotted, along with an extrapolation to $L=\infty$ (thick black line). Here $\alpha=1.5$ and $b=3$. The inset shows that the occupation of a site far away from the condensate has a subcritical distribution (i.e., with an exponential tail) when $\alpha>1$. This differs from the usual ZRP where the background fluid is known to be critical. Results are for $L=1000, \rho=10$, and $b=3$. density and the background density in the condensed phase are $\rho_{c} \approx 0.5$ and $\rho_{\mathrm{BG}} \approx 0.436$ (see Fig. 2). These deviate from the corresponding $\mathrm{MF}$ values $\rho_{c}^{(\mathrm{MF})}=1 /(b-2)=1$ and $\rho_{\mathrm{BG}}^{(\mathrm{MF})}=\rho\left(1 / w^{*}\right) \approx 0.451$ [see Eqs. (7) and (8)]. Such quantitative discrepancies result from correlations between sites which are neglected in the MF approximation.

\section{CONCLUSION}

The mechanism for the condensate drift found in this model can be summarized as follows: the spontaneous breaking of translation invariance by the formation of the condensate may induce an accumulation of particles in a nearby site. This accumulation results in a continually drifting condensate, since whenever a condensate is established on a new site, another one begins to form further ahead. This mechanism holds in a much more general setting, including when other forms $w(n)$, partially asymmetric hopping and higher dimensional lattices are considered, and more widely in other nonfactorized mass-transport models [29]. Note that the drift discussed here, in which the two most occupied sites are typically nearest neighbors, cannot occur in models with a factorized steady state, the latter being symmetric under site permutations. In this respect, our mechanism differs from that studied recently in Ref. [25], where unbounded hopping rates generate a drift (with infinite velocity) in a model whose steady state factorizes.

An important point to note is that in general the new condensate site does not have to be a neighbor of the old one. For instance, in our model (1) and (2) with $\alpha<1$, a similar analysis shows that the condensate skips every other site, as observed in Fig. 1(d) [29]. In this case, the supercritical site is site 3, rather than 2 (when the condensate is located on site 1). In principle, it may happen that there is more than one supercritical site, possibly leading to more complicated condensate drifts. It may also happen that no other site is supercritical, in which case the condensate would not drift. A precise and general classification of the conditions under which a condensate drift occurs remains an interesting open problem. However, in many specific models, a study of condensation and the condensate motion can be carried out following the mean-field procedure outlined in this paper. For instance, a recently proposed accelerated exclusion process (AEP) [33] can be analyzed in a similar fashion, yielding the phase diagram of the model and revealing that the AEP condensate drifts in the steady state [34]. It would be very interesting to explore whether the mechanism for condensate motion presented in this paper is found in other mass-transport contexts such as condensation in granular gases [1], jamming in systems of vehicular and biological traffic [11], and in models of Brownian and molecular motors [35-37].

\section{ACKNOWLEDGMENTS}

We thank Ariel Amir, Amir Bar, Or Cohen, and Tridib Sadhu for useful discussions. The support of the Israel Science Foundation (ISF) is gratefully acknowledged. 
[1] K. van der Weele, D. van der Meer, M. Versluis, and D. Lohse, Europhys. Lett. 53, 328 (2001).

[2] O. J. O’Loan, M. R. Evans, and M. E. Cates, Phys. Rev. E 58, 1404 (1998).

[3] D. Chowdhury, L. Santen, and A. Schadschneider, Phys. Rep. 329, 199 (2000).

[4] J. Kaupužs, R. Mahnke, and R. J. Harris, Phys. Rev. E 72, 056125 (2005).

[5] J. P. Bouchaud and M. Mézard, Physica A 282, 536 (2000).

[6] Z. Burda, D. Johnston, J. Jurkiewicz, M. Kaminski, M. A. Nowak, G. Papp, and I. Zahed, Phys. Rev. E 65, 026102 (2002).

[7] S. N. Majumdar, S. Krishnamurthy, and M. Barma, Phys. Rev. Lett. 81, 3691 (1998).

[8] S. N. Dorogovtsev and J. F. F. Mendes, Evolution of Networks (Oxford University Press, Oxford, 2003).

[9] M. R. Evans and T. Hanney, J. Phys. A 38, R195 (2005).

[10] S. N. Majumdar, in Exact Methods in Low-Dimensional Statistical Physics and Quantum Computing: Lecture Notes of the Les Houches Summer School July 2008, edited by J. Jacobsen et al., Vol. 89 (Oxford University Press, Oxford, 2010), arXiv:0904.4097v1.

[11] A. Schadschneider, D. Chowdhury, and K. Nishinari, Stochastic Transport in Complex Systems: From Molecules to Vehicles (Elsevier Science, Amsterdam, 2010).

[12] Y. Schwarzkopf, M. R. Evans, and D. Mukamel, J. Phys. A 41, 205001 (2008).

[13] S. Grosskinsky and G. M. Schütz, J. Stat. Phys. 132, 77 (2008).

[14] P. I. Chleboun, Ph.D. thesis, University of Warwick, 2011 (unpublished).

[15] M. R. Evans, T. Hanney, and S. N. Majumdar, Phys. Rev. Lett. 97, 010602 (2006)

[16] C. Godrèche and J. M. Luck, J. Stat. Mech. (2012) P12013.
[17] Y. Kafri, E. Levine, D. Mukamel, G. M. Schütz, and J. Török, Phys. Rev. Lett. 89, 035702 (2002).

[18] C. Godrèche and J. M. Luck, J. Phys. A 38, 7215 (2005).

[19] J. Beltrán and C. Landim, Probab. Theory Relat. Fields 152, 781 (2012).

[20] C. Landim, arXiv:1204.5987.

[21] M. J. Lighthill and G. B. Whitham, Proc. R. Soc. London A 229, 317 (1955).

[22] J. Treiterer, Ohio State University Report No. EES 278, 1975 (unpublished).

[23] O. Hirschberg, D. Mukamel, and G. M. Schütz, Phys. Rev. Lett. 103, 090602 (2009).

[24] O. Hirschberg, D. Mukamel, and G. M. Schütz, J. Stat. Mech. (2012) P08014.

[25] B. Waclaw and M. R. Evans, Phys. Rev. Lett. 108, 070601 (2012).

[26] M. R. Evans, Braz. J. Phys. 30, 42 (2000).

[27] F. Spitzer, Adv. Math. 5, 246 (1970).

[28] E. D. Andjel, Ann. Probab. 10, 525 (1982).

[29] O. Hirschberg, D. Mukamel, and G. M. Schütz (unpublished).

[30] See Supplemental Material at http://link.aps.org/supplemental/ 10.1103/PhysRevE.87.052116 for animations of the condensate drift in our model.

[31] E. Levine, D. Mukamel, and G. M. Schütz, J. Stat. Phys. 120, 759 (2005).

[32] V. Y. Chernyak, M. Chertkov, D. A. Goldberg, and K. Turitsyn, J. Stat. Phys. 140, 819 (2010).

[33] J. Dong, S. Klumpp, and R. K. P. Zia, Phys. Rev. Lett. 109, 130602 (2012).

[34] O. Hirschberg and D. Mukamel (unpublished).

[35] P. Reimann, Phys. Rep. 361, 57 (2002).

[36] P. Hänggi and F. Marchesoni, Rev. Mod. Phys. 81, 387 (2009).

[37] R. Lipowsky, Y. Chai, S. Klumpp, S. Liepelt, and M. J. I. Müller, Physica A 372, 34 (2006). 